\title{
Changes of Peel Essential Oil Composition of Four Tunisian Citrus during Fruit Maturation
}

\author{
Soumaya Bourgou, Fatma Zohra Rahali, Iness Ourghemmi, and Moufida Saïdani Tounsi
}

Laboratoire des Substances Bioactives, Centre de Biotechnologie à la Technopole de Borj-Cédria Hammam Lif 2050, Tunisia

Correspondence should be addressed to Soumaya Bourgou, soumaya_swiss@yahoo.ca

Received 24 October 2011; Accepted 15 December 2011

Academic Editor: Gifone Aguiar Rocha

Copyright ( $) 2012$ Soumaya Bourgou et al. This is an open access article distributed under the Creative Commons Attribution License, which permits unrestricted use, distribution, and reproduction in any medium, provided the original work is properly cited.

\begin{abstract}
The present work investigates the effect of ripening stage on the chemical composition of essential oil extracted from peel of four citrus: bitter orange (Citrus aurantium), lemon (Citrus limon), orange maltaise (Citrus sinensis), and mandarin (Citrus reticulate) and on their antibacterial activity. Essential oils yields varied during ripening from 0.46 to $2.70 \%$, where mandarin was found to be the richest. Forty volatile compounds were identified. Limonene (67.90-90.95\%) and 1,8-cineole (tr-14.72\%) were the most represented compounds in bitter orange oil while limonene (37.63-69.71\%), $\beta$-pinene (0.63-31.49\%), $\gamma$-terpinene $(0.04-9.96 \%)$, and $p$-cymene $(0.23-9.84 \%)$ were the highest ones in lemon. In the case of mandarin, the predominant compounds were limonene (51.81-69.00\%), 1,8-cineole (0.01-26.43\%), and $\gamma$-terpinene (2.53-14.06\%). However, results showed that orange peel oil was dominated mainly by limonene $(81.52-86.43 \%)$ during ripening. The results showed that ripening stage influenced significantly the antibacterial activity of the oils against Staphylococcus aureus, Escherichia coli, and Pseudomonas aeruginosa. This knowledge could help establish the optimum harvest date ensuring the maximum essential oil, limonene, as well as antibacterial compounds yields of citrus.
\end{abstract}

\section{Introduction}

The genus Citrus of the family Rutaceae includes several important fruits such as oranges, mandarins, limes, lemons, and grapefruits. Citrus fruits are one of the important horticultural crops, with worldwide agricultural production over 80 million tons per year [1]. Although, the fruits are mainly used for dessert, they have important economic value for their essential oils. Citrus essential oils are obtained as byproducts of the citrus processing and are the most widely used essential oils in the world. In fact, among the great variety of essential oils, citrus fruit essential oils and their major components have gained acceptance in the food industry since they have been generally recognized as safe, and many foods tolerate their presence [2].

Citrus essential oils have wide uses. Primarily, they are used as aroma flavour in many food products, including alcoholic and nonalcoholic beverages, marmalades, gelatins, sweets, soft drinks, ice creams, dairy products, candies, and cakes $[3,4]$. In pharmaceutical industries they are employed as flavoring agents to mask unpleasant tastes of drugs. Additionally, in perfumery and cosmetic, the low volatile essential oil components play an important role as head notes (e.g., in Eaux-de-Cologne and soap perfumes) [3]. Recently, other applications make use of the major compound of the oil extracted from citrus peels, limonene, as green solvent for Soxhlet extraction [5] due to its ability to solubilize fats [6].

The chemical composition of the essential oil from citrus peels has been studied and reviewed [7-12]. However, there have been few research projects focusing on volatiles during citrus fruit ripening. Dugo et al. [13] investigated the seasonal variation of the chemical composition of the essential oil extracted from the whole fruit of two cultivars of Sicilian mandarin (Citrus. deliciosa Tenore. cv Avana and Tardivo di Ciaculli) and reported a decrease of limonene level at the beginning and the end of the season. According to Vekiari et al. [14] harvesting time is a critical parameter influencing significantly the chemical compositions of the Cretan lemon peel and leaf oil. Likewise, Droby et al. [9] analysed the composition of peel essential oil of various 
citrus cultivars including sweet orange, clementine, and grapefruit at different stages of maturity and found that limonene was the predominant compound through ripening.

Although the chemical composition of peel essential oil extracted from various Tunisian citrus varieties has been studied by Hosni et al. [15], data regarding the effect of ripening on the oil chemical composition as well as the effect of ripening stage on the antibacterial activity of the citrus oils have not been reported. Therefore, the objectives of this study were to evaluate the volatile profile during the maturation of four citrus fruits, in order to understand the significance of those compounds in the ripening process of this fruits and to determine the optimal accumulation period of desirable compounds and to evaluate the antibacterial activity variation during ripening. Information on the effects of ripening on oil composition and bioactivities is crucial to optimize harvesting protocols.

\section{Materials and Methods}

2.1. Materials. Fruits of Citrus of four species: bitter orange (Citrus aurantium) cultivar Larange, lemon (Citrus limon) cultivar Beldi, orange maltaise (Citrus sinensis) cultivar Lsen asfour, mandarin (Citrus reticulate) cultivar Elarbi, were evaluated in this study. Samples were collected at three harvesting periods: stage 1 (green colour, immature), stage 2 (yellow colour; semi-mature), and stage 3 (orange colour: mature), in 2009, from Menzel Bouzelfa in the North East of Tunisia (latitude $36^{\circ} 42^{\prime} 13^{\prime} .17^{\prime \prime}$; longitude $10^{\circ} 29^{\prime} 46.93^{\prime \prime}$ ). The fruits peels including flavedo (epicarp) and albedo (mesocarp) layers were peeled off carefully and discarded.

2.1.1. Essential Oil Isolation. The fresh peels (100 g) were submitted to hydrodistillation for 120 min using a Clevengertype apparatus. This time was fixed after a kinetic survey during $30,60,90,120,150,180$, and $210 \mathrm{~min}$. The oils obtained were dried over anhydrous sodium sulphate and stored at $-20^{\circ} \mathrm{C}$ in darkness until analysed.

2.2. GC and GC-MS Analysis. Essential oils were analyzed by gas chromatography (GC) using a Hewlett-Packard 6890 apparatus (Agilent Technologies, Palo Alto, CA, USA) equipped with a flame ionization detector (FID) and an electronic pressure control (EPC) injector. A HP-Innowax capillary column (polyethylene glycol: $30 \mathrm{~m} \times 0.25 \mathrm{~mm}$ i.d., $0.25 \mu \mathrm{m}$ film thickness; Agilent Technologies, HewlettPackard, CA, USA) was used; the flow of the carrier gas $\left(\mathrm{N}_{2}\right)$ was $1.6 \mathrm{~mL} / \mathrm{min}$ and the split ratio $60: 1$. Analyses were performed using the following temperature program: oven temps isotherm at $35^{\circ} \mathrm{C}$ for $10 \mathrm{~min}$, from 35 to $205^{\circ} \mathrm{C}$ at the rate of $3^{\circ} \mathrm{C} / \mathrm{min}$, and isotherm at $205^{\circ} \mathrm{C}$ over $10 \mathrm{~min}$. Injector and detector temperature were held, respectively, at 250 and $300^{\circ} \mathrm{C}$.

2.3. Gas Chromatography-Mass Spectrometry. GC-MS analyses of essential oil volatile components were carried out on a gas chromatograph HP 5890 (II) coupled to a HP 5972 mass spectrometer (Agilent Technologies, Palo Alto, CA, USA) with electron impact ionization $(70 \mathrm{eV})$. A HP-5MS capillary column $(30 \mathrm{~m} \times 0.25 \mathrm{~mm}, 0.25 \mu \mathrm{m}$ film thickness; Agilent Technologies, Hewlett-Packard, CA, USA) was used. The column temperature was programmed to rise from $50^{\circ} \mathrm{C}$ to $240^{\circ} \mathrm{C}$ at a rate of $5^{\circ} \mathrm{C} / \mathrm{min}$. The carrier gas was helium with a flow rate of $1.2 \mathrm{~mL} / \mathrm{min}$; split ratio was $60: 1$. Scan time and mass range were $1 \mathrm{~s}$ and $40-300 \mathrm{~m} / \mathrm{z}$, respectively.

2.4. Compounds Identification. Identification of essential oil volatile compounds was based on the calculation of their retention indices $(\mathrm{RI})$ relative to $\left(\mathrm{C}_{8}-\mathrm{C}_{22}\right) n$-alkanes with those of authentic compounds available in our laboratory. Further identification was made by matching their recorded mass spectra with those stored in the Wiley/NBS mass spectral library of the GC-MS data systems and other published mass spectra [16].

2.5. Screening of Antibacterial Activity. Antibacterial activity was analyzed by the disc diffusion method [17] against three human pathogenic bacteria including Gram-positive Staphylococcus aureus (ATCC 25923), and Gram-negative bacteria Escherichia coli (ATCC 35218) and Pseudomonas aeruginosa (ATCC 27853). All bacteria were grown on Mueller Hinton plate at $30^{\circ} \mathrm{C}$ for $18-24 \mathrm{~h}$ previous inoculation onto the nutrient agar. A loop of bacteria from the agar slant stock was cultivated in nutrient broth overnight and spread with a sterile cotton swap onto Petri dishes containing $10 \mathrm{~mL}$ of API suspension medium and adjusted to the $0.5 \mathrm{McF}$ arland turbidity standards with a Densimat (BioMerieux). Sterile filter paper disks ( $6 \mathrm{~mm}$ in diameter) impregnated with $10 \mu \mathrm{L}$ of essential oil were placed on the cultured plates. After 1$2 \mathrm{~h}$ at $4^{\circ} \mathrm{C}$, the treated Petri dishes were incubated at 25 or $37^{\circ} \mathrm{C}$ for $18-24 \mathrm{~h}$. The antimicrobial activity was evaluated by measuring the diameter of the growth inhibition zone around the discs. Each experiment was carried out in triplicate, and the mean diameter of the inhibition zone was recorded.

2.6. Statistical Analyses. All data are reported as means \pm standard deviation of three samples. Statistical analysis was performed with STATISTICA. Differences were tested for significance by using the ANOVA procedure, using a significance level of $P<0.05$.

\section{Results and Discussion}

3.1. Yields of Essential Oils. The essential oils yields of four citrus peels during fruit maturation are shown in Table 1. Species and harvest time had significant effect on essential oil yield. Independently to ripening stage, mandarin exhibited the highest yield $(2.70 \%)$ followed by lemon $(1.30 \%)$ and orange $(0.74 \%)$ while bitter orange showed the smallest value of $0.46 \%$.

On the other hand, essential oil yields varied during ripening to reach maximum values during the middle stage of maturity (stage 2) for mandarin and orange while the highest lemon yield was found at the beginning of fruit 
TABLE 1: Yields (\%) of peels essential oils from four cultivars of citrus at different ripening stages.

\begin{tabular}{lcccc}
\hline $\begin{array}{l}\text { Ripening } \\
\text { stage }\end{array}$ & $\begin{array}{c}\text { Bitter } \\
\text { orange }\end{array}$ & Lemon & $\begin{array}{c}\text { Orange } \\
\text { maltaise }\end{array}$ & Mandarin \\
\hline Stage 1 & $0.23^{\mathrm{Bb}}$ & $1.30^{\mathrm{Aa}}$ & $0.13^{\mathrm{Cb}}$ & $0.22^{\mathrm{Cb}}$ \\
Stage 2 & $0.12^{\mathrm{Cd}}$ & $0.48^{\mathrm{Bc}}$ & $0.74^{\mathrm{Ab}}$ & $2.70^{\mathrm{Aa}}$ \\
Stage 3 & $0.46^{\mathrm{Ac}}$ & $0.62^{\mathrm{Bbc}}$ & $0.52^{\mathrm{Bc}}$ & $1.13^{\mathrm{Ba}}$ \\
\hline
\end{tabular}

Means of three replicates. (Values with different superscripts are significantly different at $P<0.05$ ). Caps superscripts (comparison between stages). Small superscripts (comparison between citrus species).

maturation and decreased after that. Bitter orange showed different pattern behaviour evolution from other species since the yield doubled during ripening from 0.23 at stage 1 to $0.46 \%$ at stage 3 . Vekiari et al. [14] reported a seasonal variation of the yield of lemon peel essential oil extracted from Zambetakis variety cultivated in the island of Crete with the highest value reached at the middle of the season.

Our results concerning the mature stage are in accordance with those of Hosni et al. [15] who demonstrated that Tunisian mandarin peel was the richest on essential oil compared to orange and bitter orange. However, these authors reported higher values (varying from 1.24 to $4.62 \%$ ). Such differences could be due to the effect of extraction procedure and environmental conditions. In fact, these authors used dried and ground material from citrus cultivated in Mograne region which is known to belong to the semiarid region, while in our experiment we used a fresh material collected from Menzel Bouzelfa which belongs to the humid region. Extractions parameters are known to greatly influence the essential oil yield [18, 19]; moreover, water supply during ripening was reported to influence considerably the essential oil content with an enhancement of the yield under moderate water shortage conditions [20,21].

On the other hand, the yields obtained in our study were higher than those reported in the literature; Ahmad et al. [22] reported yields varying from 0.30 to $1.21 \%$ for the four citrus varieties from Pakistan. Moreover, lower yields were reported for the mandarins from France (yield ranging from $0.05 \%$ to $0.60 \%$ ) by Lota et al. [8] and the mandarin from Colombia (yield of $0.79 \%$ ) by Blanco Tirado et al. [23].

3.2. Essential Oil Composition. Analysis of citrus peel essential oils composition showed 39 identified compounds presenting fluctuations during ripening (Table 2).

3.2.1. Bitter Orange. Analysis of the essential oil indicated that it is made essentially from monoterpenes hydrocarbons which constitute the main class during ripening varying from 71.21 to $94.61 \%$ and reaching a maximum at full maturity (Table 2). Oxygenated monoterpenes were the second class. This later was present with appreciable levels at the first and the middle stages of maturity (7.75 and $22.51 \%$ ); however, it was markedly reduced at maturity $(2.11 \%)$. Sesquiterpenes were weakly represented during the ripening.

Analysis of the volatile composition showed the predominance of limonene which level varied from 67.90 to
90.95\% during ripening, with the highest value reached at the maturity stage (Table 3 ). The relevance of limonene in mature bitter orange peel is in accordance with previous reports [15]. The essential oil was also characterized by appreciable levels of camphor $(0.17-6.37 \%)$, cis-linalool oxide $(\operatorname{tr}-3.40 \%), \alpha$-terpinene $(0.91-1.66 \%)$, and octanol $(0.02-1.59 \%)$. Moreover, the results showed that particularly 1,8 -cineole reached an important level of $(14.7 \%)$ at the semimature stage.

Despite the dominance of limonene, the low-abundant compounds are known to contribute actively to citrus aroma. Thus, camphor, which has green dry leave note [24], could mainly influence the aroma in the first stage of ripening while 1,8-cineole, characterised by a fresh and cool aroma [25], could participate actively to the citrus aroma at the middle stage. However, several minor compounds including $\alpha$-terpinene (lemon aroma) [26], $\alpha$-terpineol (green and floral-like aroma) [27], and carvacrol may influence the aroma at the mature stage.

Interestingly, when limonene showed the lowest level at the middle stage $(67.90 \%)$, several minor compounds including 1,8-cineole, terpinolene, sabinene hydrate, and linalool oxide reached their highest content. These later compounds are synthesised from a common precursor: $\alpha$ terpinyl cation [28]. Thus, at middle stage the biosynthesis of limonene is lowered in favour to other cyclic monoterpenes mainly 1,8 cineole. Biosynthesis of 1,8-cineole is thought to proceed from the a-terpinyl cation via an $\alpha$-terpineol intermediate which undergoes internal additional cyclization of the alcoholic oxygen [29].

3.2.2. Lemon. As for bitter orange, essential oil composition of lemon was dominated by monoterpenes hydrocarbons followed by oxygenated monoterpenes (Table 2). These classes represented $98.83,87.15$, and $90.43 \%$ for monoterpenes hydrocarbons and $3.92,8.72$, and $5.61 \%$ for oxygenated monoterpenes during first, middle, and mature stages, respectively.

Immature fruit presented a limonene chemotype since it constituted the predominant compound with a percentage of $68.08 \%$ (Table 3). However, at semimature stage, limonene level decreased (37.63\%) while $\beta$-pinene level (31.49\%) increased. Thus, the essential oil chemotype becomes "limonene/ $\beta$-pinene". At mature stage, limonene was found to be the most abundant $(69.71 \%)$.

Lota et al. [30] analyzed the volatile composition of peel oil of 43 taxa of lemon cultivated in France and reported different chemotypes including limonene and limonene/ $\beta$ pinene $/ \gamma$-terpinene. Besides, Flamini et al. [31] analysed the volatiles emitted by the pericarp of unripe and ripe Italian lemon fruit and found that limonene level enhanced from 65.30 at immature stage to $68.30 \%$ at mature stage. These authors concluded also that the emitted volatiles are originated from glandular structures which give an essential oil similar to fruit emissions.

On the other hand, other representative compounds in lemon oil were $\gamma$-terpinene and $p$-cymene (Table 3 ) which showed opposite behavior during ripening; in fact, $p$-cymene level decreased from $9.84 \%$ at immature stage to $0.23 \%$ at 
TABLE 2: Variations of levels (\%) of chemical classes of essential oils obtained from four cultivars of citrus at different ripening stages.

\begin{tabular}{|c|c|c|c|c|c|}
\hline Chemical classes & Ripening stage & Bitter orange & Lemon & $\begin{array}{l}\text { Orange } \\
\text { maltaise }\end{array}$ & Mandarin \\
\hline \multirow{3}{*}{ Monoterpenes hydrcarbons } & Stage 1 & $83.35^{\mathrm{Bab}}$ & $89.83^{\mathrm{Aa}}$ & $89.72^{\mathrm{Aa}}$ & $84.73^{\mathrm{Aab}}$ \\
\hline & Stage 2 & $71.21^{\mathrm{Cb}}$ & $87.15^{\mathrm{ABa}}$ & $87.12^{\mathrm{ABa}}$ & $66.45^{\mathrm{Bc}}$ \\
\hline & Stage 3 & $94.61^{\mathrm{Aa}}$ & $90.43^{\mathrm{Ab}}$ & $90.15^{\mathrm{Ab}}$ & $89.57^{\mathrm{Ab}}$ \\
\hline \multirow{3}{*}{ Oxygenated monoterpenes } & Stage 1 & $7.75^{\mathrm{Ba}}$ & $3.92^{\mathrm{Cb}}$ & $1.50^{\mathrm{Bc}}$ & $2.10^{\mathrm{Bb}}$ \\
\hline & Stage 2 & $22.51^{\mathrm{Aa}}$ & $8.72^{\mathrm{Ab}}$ & $7.15^{\mathrm{Ab}}$ & $28.68^{\mathrm{Aa}}$ \\
\hline & Stage 3 & $2.11^{\mathrm{Cc}}$ & $5.61^{\mathrm{Ba}}$ & $1.82^{\mathrm{Bd}}$ & $3.54^{\mathrm{Bb}}$ \\
\hline \multirow{3}{*}{ Sesquiterpenes hydrocarbon } & Stage 1 & $0.35^{\mathrm{Aa}}$ & $0.06^{\mathrm{Bb}}$ & $0.26^{\mathrm{Aa}}$ & $0.01^{\mathrm{Bb}}$ \\
\hline & Stage 2 & $0.44^{\mathrm{Ab}}$ & $1.28^{\mathrm{Aa}}$ & $0.28^{\mathrm{Ab}}$ & $0.08^{\mathrm{Bc}}$ \\
\hline & Stage 3 & $0.21^{\mathrm{Abc}}$ & $1.39^{\mathrm{Aa}}$ & $0.35^{\mathrm{Ab}}$ & $0.34^{\mathrm{Ab}}$ \\
\hline \multirow{3}{*}{ Oxygenated sesquiterpenes } & Stage 1 & $0.42^{\mathrm{Ab}}$ & $0.01^{\mathrm{Ac}}$ & $0.01^{\mathrm{Ac}}$ & $1.36^{\mathrm{Ba}}$ \\
\hline & Stage 2 & $0.16^{\mathrm{Bb}}$ & $0.01^{\mathrm{Ac}}$ & $0.01^{\mathrm{Ac}}$ & $0.27^{\mathrm{Ca}}$ \\
\hline & Stage 3 & $0.09^{\mathrm{Bb}}$ & $0.01^{\mathrm{Ab}}$ & $0.01^{\mathrm{Ab}}$ & $3.29^{\mathrm{Aa}}$ \\
\hline
\end{tabular}

Means of three replicates. (Values with different superscripts are significantly different at $P<0.05$ ). Caps superscripts (comparison between stages). Small superscripts (comparison between citrus species).

maturity while $\gamma$-terpinene level augmented to reach $9.96 \%$ at maturity. This is in accordance with their biosynthetic pathway, in which $\gamma$-terpinene is a precursor of $p$-cymene [32]. Further, the Germacrene D and valencene were the most abandon sesquiterpenes (Table 3 ). Minor compounds including valencene have stand out in citrus as important flavour and aroma compounds [33].

3.2.3. Orange Maltaise. Differently to bitter orange and lemon, the peel essential oil of orange fruit extracted at three stages of maturity was mainly dominated by limonene which presented a level of $86.43,81.52$, and $85.35 \%$ at immature, semimature, and mature stages, respectively (Table 3 ). The rest of compounds were weakly represented with levels lower than $1 \%$ except for the monoterpenes $\beta$-pinene, camphor and bornyl acetate. These compounds reached their maximum at semimature stage with levels of $1.80,4.81$, and $4.21 \%$, respectively. The increase of camphor and $\beta$ pinene levels suggests an activation of the related terpene synthases which catalyze their formation from the critical intermediates pinyl and bornyl cations, respectively [29]. Concerning sesquiterpenes, $\alpha$-humulene $(0.16-0.34 \%)$ followed by germacrene-D $(\operatorname{tr}-0.12 \%)$ were found to be the most represented compounds.

Our results are in accordance with that of Droby et al. [9] who analysed the composition of peel essential oil of sweet orange fruit at different stages of maturity and found that limonene was the predominant compound with percentage varying from 72.41 to $94.77 \%$. However, these authors reported a decrease of limonene level during the course of fruit maturity especially at the end of the ripening. Such difference could be attributed to both interactions between genetic (biotic) and environmental (abiotic) factors since in their study, these authors considered "Valencia" and not maltaise cultivar.

On the other hand, concerning the mature stage, Hosni et al. [15] reported a higher limonene level (96.00\%) in the peel essential oil extracted from Tunisian maltaise orange than that obtained in our study. These authors found that $\beta$-pinene was also a marked compound (1.82\%). However, camphor was not detected in their samples.

3.2.4. Mandarin. Analysis of peel oil composition of mandarin during ripening indicated that immature stage was characterized by the predominance of monoterpenes hydrocarbons where limonene $(65.37 \%)$ followed by $\gamma$-terpinene $(12.44 \%)$ were the main compounds. Moreover, borneol $(1.22 \%)$ and caryophyllene oxide $(1.18 \%)$ were the most represented oxygenated monoterpenes and sesquiterpenes, receptively (Tables 2 and 3 ).

Limonene and $\gamma$-terpinene levels decreased at semimature stage to 51.81 and $2.53 \%$, respectively. This decrease was accompanied with a concomitant increase of 1,8-cineole $(26.43 \%)$ and E- $\beta$-ocimene $(7.93 \%)$ levels. The enhancement of the biosynthesis of these compounds suggests an activation of the related synthases at semimature stage. The cyclic monoterpenes including limonene, $\gamma$-terpinene, and $\alpha$-terpineol are produced from a common intermediate: terpeiyl cation. $\alpha$-terpineol undergoes after that cyclisation to lead 1,8-cineole. On the other hand, the enhancement of the acyclic terpene $\mathrm{E}$ - $\beta$-ocimene level suggest the activation of $\mathrm{E}$ $\beta$-ocimene synthase which derives its conversion from linalyl cation [29]. Shimada et al. [34] reported that the expression of 1,8-cineole and $\mathrm{E}$ - $\beta$-ocimene genes and their related products in Satsuma mandarin was high in flower and then decreased toward mandarin fruit development. At maturity, 1,8-cineole and E- $\beta$-ocimene levels decreased. The essential oil was found to be dominated by limonene $(69.00 \%)$ followed by $\gamma$-terpinene $(14.06 \%)$. If considering only the mature stage, similar composition has been described by Lota et al. [7] who reported limonene (52.20-96.20\%) and $\gamma$-terpinene $(\operatorname{tr}-36.70 \%)$ as the main compounds of French mandarins peel oils. 
TABLE 3: Variations of chemical composition (\%) of essential oils obtained from four cultivars of citrus at different ripening stages.

\begin{tabular}{|c|c|c|c|c|c|c|c|}
\hline Volatile compounds ${ }^{A}$ & $\mathrm{RI}^{\mathrm{a}}$ & $\mathrm{RI}^{\mathrm{b}}$ & $\begin{array}{l}\text { Ripening } \\
\text { stage }\end{array}$ & Bitter orange & Lemon & $\begin{array}{l}\text { Orange } \\
\text { maltaise }\end{array}$ & Mandarin \\
\hline & & & Stage 1 & $0.05^{\mathrm{Aab}}$ & $0.16^{\mathrm{Aa}}$ & $\operatorname{tr}$ & $0.09^{\mathrm{Bab}}$ \\
\hline \multirow[t]{3}{*}{ Tricyclen } & 924 & 1014 & Stage 2 & $0.75^{\mathrm{Aa}}$ & $0.021^{\mathrm{Ab}}$ & $0.09^{\mathrm{Ab}}$ & $0.14^{\mathrm{Ab}}$ \\
\hline & & & Stage 3 & $0.0^{\mathrm{Ab}}$ & $0.02^{\mathrm{Ab}}$ & $\operatorname{tr}$ & $0.15^{\mathrm{Aa}}$ \\
\hline & & & Stage 1 & $0.14^{\mathrm{Ba}}$ & $0.38^{\mathrm{Ab}}$ & $0.37^{\mathrm{Ab}}$ & $1.31^{\mathrm{Aa}}$ \\
\hline \multirow[t]{3}{*}{$\alpha$-thujene } & 928 & 1035 & Stage 2 & $0.27^{\mathrm{ABa}}$ & $\operatorname{Tr}$ & $\operatorname{tr}$ & $0.12^{\mathrm{Bb}}$ \\
\hline & & & Stage 3 & $0.44^{\mathrm{Aa}}$ & $0.34^{\mathrm{Aa}}$ & $0.20^{\mathrm{Aa}}$ & $0.39^{\mathrm{Ba}}$ \\
\hline & & & Stage 1 & $0.13^{\mathrm{Bc}}$ & $1.29^{\mathrm{Aa}}$ & $0.41^{\mathrm{Ab}}$ & $0.03^{\mathrm{Cd}}$ \\
\hline \multirow[t]{3}{*}{$\alpha$-pinene } & 939 & 1032 & Stage 2 & $0.03^{\mathrm{Cb}}$ & $5.9^{\mathrm{Aa}}$ & $0.44^{\mathrm{Ab}}$ & $0.7^{\mathrm{Bb}}$ \\
\hline & & & Stage 3 & $0.36^{\mathrm{Ab}}$ & $1.14^{\mathrm{Aa}}$ & $0.7^{\mathrm{Aab}}$ & $1.25^{\mathrm{Aa}}$ \\
\hline & & & Stage 1 & $0.24^{\mathrm{Aab}}$ & $0.13^{\mathrm{Bb}}$ & $0.07^{\mathrm{Ab}}$ & $0.39^{\mathrm{Aa}}$ \\
\hline \multirow[t]{3}{*}{ Camphene } & 954 & 1076 & Stage 2 & $\operatorname{tr}$ & $0.9^{\mathrm{Aa}}$ & $1.22^{\mathrm{Aa}}$ & $\operatorname{tr}$ \\
\hline & & & Stage 3 & $0.02^{\mathrm{ABa}}$ & $0.03^{\mathrm{Ba}}$ & $0.06^{\mathrm{Aa}}$ & $0.09^{\mathrm{Ba}}$ \\
\hline & & & Stage 1 & $\operatorname{tr}$ & $6.48^{\mathrm{Aa}}$ & $\operatorname{tr}$ & $1.31^{\mathrm{Aa}}$ \\
\hline \multirow[t]{3}{*}{ Sabinene } & 975 & 1132 & Stage 2 & $\operatorname{tr}$ & $3.8^{\mathrm{Aa}}$ & $\operatorname{tr}$ & $0.0^{\mathrm{Ba}}$ \\
\hline & & & Stage 3 & $0.20^{\mathrm{Ab}}$ & $5.82^{\mathrm{Aa}}$ & $0.36^{\mathrm{Ab}}$ & $0.18^{\mathrm{Bb}}$ \\
\hline & & & Stage 1 & $0.11^{\mathrm{Bd}}$ & $1.06^{\mathrm{Ab}}$ & $1.54^{\mathrm{Ab}}$ & $0.43^{\mathrm{Bc}}$ \\
\hline \multirow[t]{3}{*}{$\beta$-pinene } & 980 & 1118 & Stage 2 & $0.07^{\mathrm{Ba}}$ & $31.49^{\mathrm{Aa}}$ & $1.80^{\mathrm{Aa}}$ & $0.06^{\mathrm{Ba}}$ \\
\hline & & & Stage 3 & $0.38^{\mathrm{Ab}}$ & $0.63^{\mathrm{Aa}}$ & $0.97^{\mathrm{Ab}}$ & $0.75^{\mathrm{Ab}}$ \\
\hline & & & Stage 1 & $0.09^{\mathrm{Ab}}$ & $1.54^{\mathrm{Aa}}$ & $\operatorname{tr}$ & $1.59^{\mathrm{Aa}}$ \\
\hline \multirow[t]{3}{*}{ Myrcene } & 991 & 1174 & Stage 2 & $0.48^{\mathrm{Aa}}$ & $\operatorname{Tr}$ & $\operatorname{tr}$ & $\operatorname{tr}$ \\
\hline & & & Stage 3 & $0.07^{\mathrm{Aa}}$ & $0.99^{\mathrm{Ba}}$ & $0.71^{\mathrm{Aa}}$ & $0.98^{\mathrm{Aa}}$ \\
\hline & & & Stage 1 & $\operatorname{tr}$ & $\operatorname{Tr}$ & $\operatorname{tr}$ & $\operatorname{tr}$ \\
\hline \multirow[t]{3}{*}{$\Delta$-3-Carene } & 1011 & 1159 & Stage 2 & $\operatorname{tr}$ & $\operatorname{Tr}$ & $\operatorname{tr}$ & $\operatorname{tr}$ \\
\hline & & & Stage 3 & $0.01^{\mathrm{Aa}}$ & $\operatorname{Tr}$ & $0.05^{\mathrm{Aa}}$ & $0.03^{\mathrm{Aa}}$ \\
\hline & & & Stage 1 & $1.23^{\mathrm{Aa}}$ & $0.24^{\mathrm{Bb}}$ & $\operatorname{tr}$ & $0.30^{\mathrm{Bb}}$ \\
\hline \multirow[t]{3}{*}{$\alpha$-Terpinene } & 1018 & 1188 & Stage 2 & $0.91^{\mathrm{Aa}}$ & $\operatorname{Tr}$ & $\operatorname{tr}$ & $1.52^{\mathrm{Aa}}$ \\
\hline & & & Stage 3 & $1.66^{\mathrm{Aa}}$ & $1.05^{\mathrm{Aa}}$ & $0.93^{\mathrm{Aa}}$ & $0.73^{\mathrm{Ba}}$ \\
\hline & & & Stage 1 & $\operatorname{tr}$ & $9.84^{\mathrm{Aa}}$ & $0.25^{\mathrm{Ab}}$ & $0.63^{\mathrm{Aa}}$ \\
\hline \multirow[t]{3}{*}{ p-Cymene } & 1026 & 1280 & Stage 2 & $0.09^{\mathrm{Aab}}$ & $2.21^{\mathrm{Ba}}$ & $0.27^{\mathrm{Aab}}$ & $0.68^{\mathrm{Aa}}$ \\
\hline & & & Stage 3 & $0.08^{\mathrm{Ac}}$ & $0.23^{\mathrm{Bb}}$ & $0.21^{\mathrm{Abc}}$ & $0.7^{\mathrm{Aa}}$ \\
\hline & & & Stage 1 & $80.54^{\mathrm{Aa}}$ & $68.08^{\mathrm{Ab}}$ & $86.43^{\mathrm{Aa}}$ & $65.37^{\mathrm{Ab}}$ \\
\hline \multirow[t]{3}{*}{ Limonene } & 1030 & 1203 & Stage 2 & $67.90^{\mathrm{ABab}}$ & $37.63^{\mathrm{Bb}}$ & $81.52^{\mathrm{Aa}}$ & $51.81^{\mathrm{Aab}}$ \\
\hline & & & Stage 3 & $90.95^{\mathrm{Aa}}$ & $69.71^{\mathrm{Ab}}$ & $85.35^{\mathrm{Aa}}$ & $69.00^{\mathrm{Ab}}$ \\
\hline & & & Stage 1 & $\operatorname{tr}$ & $0.51^{\mathrm{ABa}}$ & $\operatorname{tr}$ & $0.01^{\mathrm{Bb}}$ \\
\hline \multirow[t]{3}{*}{ 1.8-Cineole } & 1033 & 1193 & Stage 2 & $14.72^{\mathrm{Aab}}$ & $0.82^{\mathrm{Ab}}$ & $\operatorname{tr}$ & $26.43^{\mathrm{Aa}}$ \\
\hline & & & Stage 3 & $0.30^{\mathrm{Aa}}$ & $\operatorname{tr}$ & $\operatorname{tr}$ & $0.18^{\mathrm{Bb}}$ \\
\hline & & & Stage 1 & $0.5^{\mathrm{Ab}}$ & $0.14^{\mathrm{Bc}}$ & $\operatorname{tr}$ & $0.77^{\mathrm{Ab}}$ \\
\hline \multirow[t]{3}{*}{$E$ - $\beta$-Ocimene } & 1050 & 1266 & Stage 2 & $0.09^{\mathrm{Bab}}$ & $\operatorname{tr}$ & $0.09^{\mathrm{Aa}}$ & $7.93^{\mathrm{Aa}}$ \\
\hline & & & Stage 3 & $0.02^{\mathrm{Bb}}$ & $0.5^{\text {Aab }}$ & $0.03^{\mathrm{Ab}}$ & $1.05^{\mathrm{Aa}}$ \\
\hline & & & Stage 1 & $0.14^{\mathrm{Ab}}$ & $0.04^{\mathrm{Bb}}$ & $\operatorname{tr}$ & $12.44^{\mathrm{Aa}}$ \\
\hline \multirow[t]{3}{*}{$\gamma$-terpinene } & 1058 & 1255 & Stage 2 & $0.21^{\mathrm{Aa}}$ & $5.12^{\mathrm{ABa}}$ & $0.34^{\mathrm{Aa}}$ & $2.53^{\mathrm{Ba}}$ \\
\hline & & & Stage 3 & $0.31^{\mathrm{Ac}}$ & $9.96^{\mathrm{Ab}}$ & $0.43^{\mathrm{Ac}}$ & $14.06^{\mathrm{Aa}}$ \\
\hline & & & Stage 1 & $\operatorname{tr}$ & $0.024^{\mathrm{Bb}}$ & $0.37^{\mathrm{Aa}}$ & $0.04^{\mathrm{Aa}}$ \\
\hline \multirow[t]{3}{*}{ cis-Sabinene hydrate } & 1062 & 1550 & Stage 2 & $0.18^{\mathrm{Aa}}$ & $0.05^{\mathrm{Aa}}$ & $0.26^{\mathrm{Aa}}$ & $0.24^{\mathrm{Aa}}$ \\
\hline & & & Stage 3 & $0.09^{\mathrm{Bab}}$ & $\operatorname{tr}$ & $0.14^{\mathrm{Aa}}$ & $0.04^{\mathrm{Abc}}$ \\
\hline & & & Stage 1 & $0.05^{\mathrm{Aa}}$ & $\operatorname{tr}$ & $\operatorname{tr}$ & $0.03^{\mathrm{Ba}}$ \\
\hline \multirow[t]{2}{*}{ Octanol } & 1070 & 1546 & Stage 2 & $1.59^{\mathrm{Aa}}$ & $\operatorname{tr}$ & $0.07^{\mathrm{Aa}}$ & $1.41^{\mathrm{Aa}}$ \\
\hline & & & Stage 3 & $0.02^{\mathrm{Aab}}$ & $\operatorname{tr}$ & $\operatorname{tr}$ & $0.05^{\mathrm{Ba}}$ \\
\hline
\end{tabular}


TABle 3: Continued.

\begin{tabular}{|c|c|c|c|c|c|c|c|}
\hline Volatile compounds ${ }^{\mathrm{A}}$ & $\mathrm{RI}^{\mathrm{a}}$ & $\mathrm{RI}^{\mathrm{b}}$ & $\begin{array}{l}\text { Ripening } \\
\text { stage }\end{array}$ & Bitter orange & Lemon & $\begin{array}{l}\text { Orange } \\
\text { maltaise }\end{array}$ & Mandarin \\
\hline \multirow{3}{*}{ cis-Linalool oxide } & \multirow{3}{*}{1074} & \multirow{3}{*}{1450} & Stage 1 & $\operatorname{tr}$ & $\operatorname{tr}$ & $\operatorname{tr}$ & $\operatorname{tr}$ \\
\hline & & & Stage 2 & $3.40^{\mathrm{Aa}}$ & $0.49^{\mathrm{Ab}}$ & $0.82^{\mathrm{Aab}}$ & $0.36^{\mathrm{Ab}}$ \\
\hline & & & Stage 3 & $0.09^{\mathrm{Bb}}$ & $0.0^{\mathrm{Bd}}$ & $0.03^{\mathrm{Ac}}$ & $0.14^{\mathrm{Ba}}$ \\
\hline \multirow{3}{*}{ Terpinolene } & \multirow{3}{*}{1092} & \multirow{3}{*}{1290} & Stage 1 & $0.16^{\mathrm{Ab}}$ & $0.42^{\mathrm{Aa}}$ & $0.25^{\mathrm{Ab}}$ & $0.024^{\mathrm{Cc}}$ \\
\hline & & & Stage 2 & $0.22^{\mathrm{Aa}}$ & $\operatorname{tr}$ & $1.07^{\mathrm{Aa}}$ & $0.7^{\mathrm{Aa}}$ \\
\hline & & & Stage 3 & $0.02^{\mathrm{Ab}}$ & $\operatorname{tr}$ & $\operatorname{tr}$ & $0.17^{\mathrm{Ba}}$ \\
\hline \multirow{3}{*}{ Linalool } & \multirow{3}{*}{1098} & \multirow{3}{*}{1553} & Stage 1 & $0.03^{\mathrm{Ab}}$ & $0.0^{\mathrm{Cc}}$ & $0.12^{\mathrm{Aa}}$ & $\operatorname{tr}$ \\
\hline & & & Stage 2 & $\operatorname{tr}$ & $1.59^{\mathrm{Aa}}$ & $0.54^{\mathrm{Ab}}$ & $0.09^{\mathrm{Ab}}$ \\
\hline & & & Stage 3 & $0.1^{\mathrm{Aa}}$ & $0.65^{\mathrm{Ba}}$ & $0.13^{\mathrm{Aa}}$ & $0.67^{\mathrm{Aa}}$ \\
\hline \multirow{3}{*}{ Nonanal } & \multirow{3}{*}{1102} & \multirow{3}{*}{1400} & Stage 1 & $0.12^{\mathrm{Aa}}$ & $\operatorname{tr}$ & $0.21^{\mathrm{Aa}}$ & $\operatorname{tr}$ \\
\hline & & & Stage 2 & $\operatorname{tr}$ & $\operatorname{tr}$ & $0.03^{\mathrm{Ba}}$ & $0.07^{\mathrm{Aa}}$ \\
\hline & & & Stage 3 & $0.18^{\mathrm{Aa}}$ & $\operatorname{tr}$ & $0.01^{\mathrm{Bb}}$ & $0.05^{\mathrm{Ab}}$ \\
\hline \multirow{3}{*}{$\alpha$-Thujone } & \multirow{3}{*}{1115} & \multirow{3}{*}{1413} & Stage 1 & $0.35^{\mathrm{Aa}}$ & $\operatorname{tr}$ & $0.06^{\mathrm{Ab}}$ & $0.05^{\mathrm{Ab}}$ \\
\hline & & & Stage 2 & $\operatorname{tr}$ & $\operatorname{tr}$ & $\operatorname{tr}$ & $0.03^{\mathrm{Aa}}$ \\
\hline & & & Stage 3 & $0.09^{\text {Aab }}$ & $\operatorname{tr}$ & $\operatorname{tr}$ & $0.17^{\mathrm{Aa}}$ \\
\hline \multirow{3}{*}{ Camphor } & \multirow{3}{*}{1144} & \multirow{3}{*}{1550} & Stage 1 & $6.37^{\mathrm{Aa}}$ & $\operatorname{tr}$ & $\operatorname{tr}$ & $\operatorname{tr}$ \\
\hline & & & Stage 2 & $1.43^{\mathrm{Aa}}$ & $0.32^{\mathrm{ABa}}$ & $4.81^{\mathrm{Aa}}$ & $0.039^{\mathrm{Aa}}$ \\
\hline & & & Stage 3 & $0.17^{\mathrm{Ab}}$ & $0.58^{\mathrm{Aa}}$ & $0.17^{\mathrm{Ab}}$ & $0.35^{\text {Aab }}$ \\
\hline \multirow{3}{*}{ Borneol } & \multirow{3}{*}{1165} & \multirow{3}{*}{1719} & Stage 1 & $0.13^{\mathrm{Ab}}$ & $0.33^{\mathrm{Bb}}$ & $0.14^{\mathrm{Ab}}$ & $1.22^{\mathrm{Aa}}$ \\
\hline & & & Stage 2 & $0.16^{\mathrm{Aa}}$ & $0.65^{\mathrm{Ba}}$ & $0.7^{\mathrm{Aa}}$ & $0.04^{\mathrm{Ba}}$ \\
\hline & & & Stage 3 & $0.08^{\mathrm{Ab}}$ & $2.54^{\mathrm{Aa}}$ & $0.3^{\mathrm{Ab}}$ & $0.04^{\mathrm{Bb}}$ \\
\hline \multirow{3}{*}{ Terpinene-4-ol } & & & Stage 1 & $0.05^{\mathrm{Aa}}$ & $\operatorname{tr}$ & $\operatorname{tr}$ & $\operatorname{tr}$ \\
\hline & 1178 & 1611 & Stage 2 & $0.54^{\mathrm{Aa}}$ & $1.28^{\mathrm{Aa}}$ & $0.041^{\mathrm{Ba}}$ & $0.026^{\mathrm{Aa}}$ \\
\hline & & & Stage 3 & $0.01^{\mathrm{Ab}}$ & $\operatorname{tr}$ & $0.26^{\mathrm{Aa}}$ & $0.01^{\mathrm{Ab}}$ \\
\hline & & & Stage 1 & $0.32^{\mathrm{Ab}}$ & $1.7^{\mathrm{Aa}}$ & $\operatorname{tr}$ & $0.05^{\mathrm{Ac}}$ \\
\hline$\alpha$-Terpineol & 1189 & 1709 & Stage 2 & $0.07^{\mathrm{Aa}}$ & $0.93^{\mathrm{Aa}}$ & $0.04^{\mathrm{Ba}}$ & $0.11^{\mathrm{Aa}}$ \\
\hline & & & Stage 3 & $0.35^{\mathrm{Aa}}$ & $1.22^{\mathrm{Aa}}$ & $0.52^{\mathrm{Aa}}$ & $1.25^{\mathrm{Aa}}$ \\
\hline & & & Stage 1 & $0.27^{\mathrm{Ab}}$ & $0.29^{\mathrm{Ab}}$ & $0.13^{\mathrm{Ac}}$ & $0.52^{\mathrm{Aa}}$ \\
\hline cis-Dihydrocarvone & 1197 & 1645 & Stage 2 & $0.28^{\mathrm{Aa}}$ & $\operatorname{tr}$ & $0.1^{\mathrm{Aa}}$ & $0.02^{\mathrm{Ca}}$ \\
\hline & & & Stage 3 & $0.03^{\mathrm{Ab}}$ & $\operatorname{tr}$ & $0^{\mathrm{Bc}}$ & $0.057^{\mathrm{Ba}}$ \\
\hline & & & Stage 1 & $\operatorname{tr}$ & $0.04^{\mathrm{Ab}}$ & $\operatorname{tr}$ & $0.07^{\mathrm{Aa}}$ \\
\hline Citronellol & 1226 & 1773 & Stage 2 & $0.07^{\mathrm{Aa}}$ & $\operatorname{tr}$ & $\operatorname{tr}$ & $0.01^{\mathrm{Ab}}$ \\
\hline & & & Stage 3 & $0.03^{\mathrm{ABab}}$ & $\operatorname{tr}$ & $0.11^{\mathrm{Aa}}$ & $0.07^{\text {Aab }}$ \\
\hline & & & Stage 1 & $0.09^{\mathrm{Ab}}$ & $0.24^{\mathrm{Aa}}$ & $\operatorname{tr}$ & $\operatorname{tr}$ \\
\hline Nerol & 1228 & 1797 & Stage 2 & $0.03^{\mathrm{Aa}}$ & $\operatorname{tr}$ & $\operatorname{tr}$ & $\operatorname{tr}$ \\
\hline & & & Stage 3 & $0.12^{\mathrm{Aa}}$ & $\operatorname{tr}$ & $\operatorname{tr}$ & $0.06^{\mathrm{Ab}}$ \\
\hline & & & Stage 1 & $0.07^{\mathrm{Ab}}$ & $0.78^{\mathrm{ABa}}$ & $0.79^{\mathrm{Aa}}$ & $0.0^{\mathrm{Ab}}$ \\
\hline Linalyl acetate & 1257 & 1556 & Stage 2 & $0.09^{\mathrm{Ab}}$ & $2.61^{\mathrm{Aa}}$ & $\operatorname{tr}$ & $0.09^{\mathrm{Ab}}$ \\
\hline & & & Stage 3 & $0.01^{\mathrm{Aa}}$ & $\operatorname{tr}$ & $0.10^{\mathrm{Ba}}$ & $0.11^{\mathrm{Aa}}$ \\
\hline & & & Stage 1 & $\operatorname{tr}$ & $\operatorname{tr}$ & $\operatorname{tr}$ & $0.14^{\mathrm{Aa}}$ \\
\hline Bornyl acetate & 1295 & 1577 & Stage 2 & $\operatorname{tr}$ & $\operatorname{tr}$ & $1.45^{\mathrm{Aa}}$ & $\operatorname{tr}$ \\
\hline & & & Stage 3 & $\operatorname{tr}$ & $0.01^{\mathrm{Aa}}$ & $4.21^{\mathrm{Aa}}$ & $0.07^{\mathrm{Ba}}$ \\
\hline & & & Stage 1 & $\operatorname{tr}$ & $\operatorname{tr}$ & $0.22^{\mathrm{Aa}}$ & $0.12^{\mathrm{Ab}}$ \\
\hline 30 Carvacrol & 1302 & 2239 & Stage 2 & $\operatorname{tr}$ & $\operatorname{tr}$ & $\operatorname{tr}$ & $\operatorname{tr}$ \\
\hline & & & Stage 3 & $0.69^{\mathrm{Aa}}$ & $0.03^{\mathrm{Ab}}$ & $0.08^{\mathrm{Bb}}$ & $0.14^{\mathrm{Ab}}$ \\
\hline
\end{tabular}


TABle 3: Continued.

\begin{tabular}{|c|c|c|c|c|c|c|c|}
\hline Volatile compounds ${ }^{\mathrm{A}}$ & $\mathrm{RI}^{\mathrm{a}}$ & $\mathrm{RI}^{\mathrm{b}}$ & $\begin{array}{l}\text { Ripening } \\
\text { stage }\end{array}$ & Bitter orange & Lemon & $\begin{array}{l}\text { Orange } \\
\text { maltaise }\end{array}$ & Mandarin \\
\hline \multirow{3}{*}{$\alpha$-Terpinyl acetate } & \multirow{3}{*}{1344} & \multirow{3}{*}{1706} & Stage 1 & $1.4^{\mathrm{Aa}}$ & $\operatorname{tr}$ & $\operatorname{tr}$ & $0.02^{\mathrm{Ab}}$ \\
\hline & & & Stage 2 & $0.11^{\mathrm{Bab}}$ & $0.15^{\text {Aab }}$ & $\operatorname{tr}$ & $0.20^{\mathrm{Aa}}$ \\
\hline & & & Stage 3 & $\operatorname{tr}$ & $\operatorname{tr}$ & $\operatorname{tr}$ & $0.72^{\mathrm{Aa}}$ \\
\hline \multirow{3}{*}{ Geranyl acetate } & \multirow{3}{*}{1383} & \multirow{3}{*}{1765} & Stage 1 & $\operatorname{tr}$ & $\operatorname{tr}$ & $\operatorname{tr}$ & $\operatorname{tr}$ \\
\hline & & & Stage 2 & $0.11^{\mathrm{Aa}}$ & $\operatorname{tr}$ & $\operatorname{tr}$ & $0.02^{\mathrm{Bab}}$ \\
\hline & & & Stage 3 & $0.02^{\mathrm{Ac}}$ & $0.56^{\mathrm{Aa}}$ & $0.10^{\mathrm{Ac}}$ & $0.24^{\mathrm{Ab}}$ \\
\hline \multirow{3}{*}{$\alpha$-Humulene } & \multirow{3}{*}{1454} & \multirow{3}{*}{1687} & Stage 1 & $0.047^{\mathrm{Bb}}$ & $0.04^{\mathrm{Ab}}$ & $0.19^{\mathrm{ABa}}$ & $\operatorname{tr}$ \\
\hline & & & Stage 2 & $0.03^{\mathrm{Bb}}$ & $0.02^{\mathrm{Ab}}$ & $0.16^{\mathrm{Ba}}$ & $0.01^{\mathrm{Bb}}$ \\
\hline & & & Stage 3 & $0.13^{\mathrm{Ab}}$ & $\operatorname{tr}$ & $0.34^{\mathrm{Aa}}$ & $0.03^{\mathrm{Ac}}$ \\
\hline \multirow{3}{*}{ Germacrene D } & \multirow{3}{*}{1480} & \multirow{3}{*}{1726} & Stage 1 & $0.10^{\mathrm{Ba}}$ & $0.019^{\mathrm{Bc}}$ & $0.07^{\mathrm{Ab}}$ & $\operatorname{tr}$ \\
\hline & & & Stage 2 & $0.25^{\mathrm{Aab}}$ & $0.89^{\mathrm{ABa}}$ & $0.12^{\mathrm{Ab}}$ & $0.03^{\mathrm{Bb}}$ \\
\hline & & & Stage 3 & $0.04^{\mathrm{Cb}}$ & $1.35^{\mathrm{Aa}}$ & $\operatorname{tr}$ & $0.15^{\mathrm{Ab}}$ \\
\hline \multirow{3}{*}{ Valencene } & \multirow{3}{*}{1495} & \multirow{3}{*}{1740} & Stage 1 & $0.2^{\mathrm{Aa}}$ & $\operatorname{tr}$ & $\operatorname{tr}$ & $\operatorname{tr}$ \\
\hline & & & Stage 2 & $0.16^{\mathrm{Aa}}$ & $0.37^{\mathrm{Aa}}$ & $\operatorname{tr}$ & $0.04^{\mathrm{ABa}}$ \\
\hline & & & Stage 3 & $0.04^{\mathrm{Bb}}$ & $0.03^{\mathrm{Ab}}$ & $\operatorname{tr}$ & $0.16^{\mathrm{Aa}}$ \\
\hline \multirow{3}{*}{ Spathulenol } & \multirow{3}{*}{1577} & \multirow{3}{*}{2121} & Stage 1 & $0.21^{\mathrm{Ab}}$ & $\operatorname{tr}$ & $\operatorname{tr}$ & $0.17^{\mathrm{Ba}}$ \\
\hline & & & Stage 2 & $\operatorname{tr}$ & $\operatorname{tr}$ & $\operatorname{tr}$ & $0.21^{\mathrm{Ba}}$ \\
\hline & & & Stage 3 & $\operatorname{tr}$ & $\operatorname{tr}$ & $\operatorname{tr}$ & $2.5^{\mathrm{Aa}}$ \\
\hline \multirow{3}{*}{ Caryophyllene oxide } & \multirow{3}{*}{1580} & \multirow{3}{*}{2008} & Stage 1 & $0.21^{\mathrm{Ab}}$ & $\operatorname{tr}$ & $\operatorname{tr}$ & $1.18^{\mathrm{Aa}}$ \\
\hline & & & Stage 2 & $0.15^{\mathrm{Aa}}$ & $\operatorname{tr}$ & $\operatorname{tr}$ & $\operatorname{tr}$ \\
\hline & & & Stage 3 & $0.08^{\mathrm{Aa}}$ & $\operatorname{tr}$ & $\operatorname{tr}$ & $\operatorname{tr}$ \\
\hline \multirow{3}{*}{ 2Z.6E-Farnesol } & \multirow{3}{*}{1724} & \multirow{3}{*}{2351} & Stage 1 & $\operatorname{tr}$ & $\operatorname{tr}$ & $\operatorname{tr}$ & $\operatorname{tr}$ \\
\hline & & & Stage 2 & $\operatorname{tr}$ & $\operatorname{tr}$ & $\operatorname{tr}$ & $0.06^{\mathrm{Ba}}$ \\
\hline & & & Stage 3 & $\operatorname{tr}$ & $\operatorname{tr}$ & $\operatorname{tr}$ & $0.79^{\mathrm{Aa}}$ \\
\hline \multirow{3}{*}{ NI } & \multirow{3}{*}{-} & \multirow{3}{*}{-} & Stage 1 & $4.47^{\mathrm{Ab}}$ & $4.70^{\mathrm{Ab}}$ & $1.46^{\mathrm{Bc}}$ & $5.67^{\mathrm{Aa}}$ \\
\hline & & & Stage 2 & $5.53^{\mathrm{Aa}}$ & $1.55^{\mathrm{Bc}}$ & $2.70^{\mathrm{Ab}}$ & $1.21^{\mathrm{Bc}}$ \\
\hline & & & Stage 3 & $0.45^{\mathrm{Bb}}$ & $1.85^{\mathrm{Ba}}$ & $0.37^{\mathrm{Cb}}$ & $0.12^{\mathrm{Bb}}$ \\
\hline
\end{tabular}

Means of three replicates. (Values with different superscripts are significantly different at $P<0.05$ ). Caps superscripts (comparison between stages). Small superscripts (comparison between citrus species).

${ }^{\mathrm{A}}$ Components are listed in order of elution in apolar column (HP-5).

$\mathrm{RI}^{\mathrm{a}} \cdot \mathrm{RI}^{\mathrm{b}}$ : retention indices calculated using, respectively, an apolar column (HP-5) and polar column (HPInnowax);

NI: non identified.

TABLE 4: Antibacterial activity of peel essential oils of four cultivars of citrus at different ripening stages against three human pathogenic bacteria.

\begin{tabular}{|c|c|c|c|c|c|c|}
\hline \multirow{2}{*}{ Bacteria strains } & \multicolumn{6}{|c|}{ Diameter of inhibition zone including the disc ( $\mathrm{mm})$} \\
\hline & Ripening stage & Bitter orange & Lemon & Orange maltaise & Mandarin & Gentamicin \\
\hline \multirow{3}{*}{ E. coli } & Stage 1 & $17.30^{\mathrm{Ab}}$ & $26.60^{\mathrm{Aa}}$ & na & $26.00^{\mathrm{Aa}}$ & \multirow{3}{*}{27} \\
\hline & Stage 2 & na & na & $11.30^{\mathrm{Ba}}$ & $1.00^{\mathrm{Cb}}$ & \\
\hline & Stage 3 & $14.00^{\mathrm{Bab}}$ & $17.30^{\mathrm{Ba}}$ & $17.30^{\mathrm{Aa}}$ & $16.00^{\mathrm{Ba}}$ & \\
\hline \multirow{3}{*}{ S. aureus } & Stage 1 & $16.00^{\mathrm{Aa}}$ & $3.53^{\mathrm{Cb}}$ & na & $1.30^{\mathrm{Bb}}$ & \multirow{3}{*}{22} \\
\hline & Stage 2 & $6.00^{\mathrm{Ba}}$ & na & $1.36^{\mathrm{Bb}}$ & $1.06^{\mathrm{Bb}}$ & \\
\hline & Stage 3 & $14.00^{\mathrm{Ac}}$ & $16.60^{\mathrm{bc}}$ & $13.30^{\mathrm{Ac}}$ & $20.00^{\mathrm{Aa}}$ & \\
\hline \multirow{3}{*}{ P. aeruginosa } & Stage 1 & na & na & na & na & \multirow{3}{*}{16} \\
\hline & Stage 2 & na & na & na & na & \\
\hline & Stage 3 & $8.00^{\mathrm{b}}$ & $13.30^{\mathrm{a}}$ & $11.30^{\mathrm{a}}$ & na & \\
\hline
\end{tabular}

Means of three replicates. (Values with different superscripts are significantly different at $P<0.05$ ). Caps superscripts (comparison between stages). Small superscripts (comparison between citrus species). Na: not active. 
3.3. Antibacterial Activity. The antibacterial activity of citrus essential oils was tested against human pathogenic bacteria. The results presented in Table 4 showed great differences in the activity between citrus species and during ripening stages. The oils were effective against Gram (+) and Gram $(-)$ bacteria, with a major activity against $S$. aureus and $E$. coli. Bitter orange, lemon, and orange were effective against $P$. aeruginosa only at maturity while mandarin oil remained inactive against this strain. In accordance with our finding, Espina et al. [12] reported no activity of the peel oil extracted from mature mandarin fruit against $P$. aeruginosa. On the other hand, lemon and mandarin essential oils extracted from immature fruit exhibited the highest antibacterial activity against E. coli which was comparable of positive control activity. In the case of $S$. aureus, the oils were mostly active at mature stage with mandarin oil showing the highest antibacterial activity.

The antimicrobial activity of essential oils is believed to be associated with phytochemical components especially monoterpenes [35] which diffuse into and damage cell membrane structures. However, the differences on the oils activity found in our experiment between ripening stages may be related to the modifications of the oils composition during fruit maturation. For the four citrus species, the variations of the activity were not correlated to that of the major compound level: limonene. This is in agreement with literature data where limonene is a weak antibacterial compound [36]. Moreover, the inhibitory activity of an essential oil is known to result from a complex interaction between its different constituents, which may produce additive, synergistic, or antagonistic effects, even for those present at low concentrations. Thus, at immature stage, antibacterial compounds including camphor [37] and $\alpha$-thujone [38] in bitter orange, $\alpha$-terpineol [39] and nerol in lemon [40], and borneol [37] and caryophyllene oxide [41] in mandarin may be involved in the found activities of the corresponding oils.

At semimature stage, the higher antibacterial capacity of orange oil compared to the citrus oils could be linked to the presence of camphor at appreciable level (4.81\%). Moreover, borneol could be involved in the found activity since this monoterpene alcohol was reported to exhibit moderate antibacterial activity against $S$. aureus and E. coli but to remain inactive against $P$. aeruginosa [42]. Our results demonstrated also an important increase of 1,8 cineole in bitter orange and mandarin oils. Literature data dealing with the activity of 1,8 cineole are contrasting; Randrianarivelo et al. [43] reported that this ether is effective against $\operatorname{Gram}(+)$ and $\operatorname{Gram}(-)$ bacteria including E. coli and S. Aureus; however, Hussain et al. [44] found that 1,8 cineole remained inactive against $P$. aeruginosa and E. coli. The low activity of the bitter orange and mandarin oils at the semimature stage in spite of a high level of 1,8 cineole suggests that this compound is inactive.

The activity of the four oils extracted from mature fruits could mainly be due to the presence of the phenolic compound carvacrol. This later is a well-known antibacterial compound acting at low concentration [38]. Moreover, mandarin was distinguished from other citrus species by the presence of farnesol which was reported to be highly effective against $S$. aureus [45] while the activity of orange oil could be ascribed to the enhancement of humulene percentage, which was reported to be moderately active [46].

\section{Conclusions}

This study revealed that the ripening stage affects significantly the yield and the composition of the examined Tunisian citrus. Immature stage offered the maximum yield for lemon while semimature fruit was the best ripening stage for mandarin and orange maltaise. In the case of bitter orange, maturity was the best stage. The oils chemical compositions and the antibacterial activity changed during ripening, and the maximum levels of the most abundant volatile compounds identified were dependent on maturity stage. For the four citrus, the highest limonene level was reached already at immature stage which suggests that at least in the case of lemon and for economic purposes, fruits could be harvested at immaturity in order to obtain essential oil with high yield and limonene content.

\section{References}

[1] F. R. Marín, C. Soler-Rivas, O. Benavente-García, J. Castillo, and J. A. Pérez-Alvarez, "By-products from different citrus processes as a source of customized functional fibres," Food Chemistry, vol. 100, no. 2, pp. 736-741, 2007.

[2] K. Fisher and C. Phillips, "Potential antimicrobial uses of essential oils in food: is citrus the answer?" Trends in Food Science and Technology, vol. 19, no. 3, pp. 156-164, 2008.

[3] B. Steuer, H. Schulz, and E. Läger, "Classification and analysis of citrus oils by NIR spectroscopy," Food Chemistry, vol. 72, no. 1, pp. 113-117, 2001.

[4] H. Nguyen, E. M. Campi, W. Roy Jackson, and A. F. Patti, "Effect of oxidative deterioration on flavour and aroma components of lemon oil," Food Chemistry, vol. 112, no. 2, pp. 388-393, 2009.

[5] M. Virot, V. Tomao, C. Ginies, F. Visinoni, and F. Chemat, "Green procedure with a green solvent for fats and oils" determination. Microwave-integrated Soxhlet using limonene followed by microwave Clevenger distillation," Journal of Chromatography A, vol. 1196-1197, no. 1-2, pp. 147-152, 2008.

[6] P. K. Mamidipally and S. X. Liu, "First approach on rice bran oil extraction using limonene," European Journal of Lipid Science and Technology, vol. 106, no. 2, pp. 122-125, 2004.

[7] M. L. Lota, D. De Rocca Serra, F. Tomi, and J. Casanova, "Chemical variability of peel and leaf essential oils of mandarins from Citrus reticulata Blanco," Biochemical Systematics and Ecology, vol. 28, no. 1, pp. 61-78, 2000.

[8] M. L. Lota, D. De Rocca Serra, F. Tomi, and J. Casanova, "Chemical variability of peel and leaf essential oils of 15 species of mandarins," Biochemical Systematics and Ecology, vol. 29, no. 1, pp. 77-104, 2001.

[9] S. Droby, A. Eick, D. Macarisin et al., "Role of citrus volatiles in host recognition, germination and growth of Penicillium digitatum and Penicillium italicum," Postharvest Biology and Technology, vol. 49, no. 3, pp. 386-396, 2008.

[10] M. Chutia, P. Deka Bhuyan, M. G. Pathak, T. C. Sarma, and P. Boruah, "Antifungal activity and chemical composition of Citrus reticulata Blanco essential oil against phytopathogens 
from North East India," LWT_Food Science and Technology, vol. 42, no. 3, pp. 777-780, 2009.

[11] M. Sawamura, N. Thi Minh Tu, Y. Onishi, E. Ogawa, and H. S. Choi, "Characteristic odor components of Citrus reticulata Blanco (ponkan) cold-pressed oil," Bioscience, Biotechnology and Biochemistry, vol. 68, no. 8, pp. 1690-1697, 2004.

[12] L. Espina, M. Somolinos, S. Lorán, P. Conchello, D. García, and R. Pagán, "Chemical composition of commercial citrus fruit essential oils and evaluation of their antimicrobial activity acting alone or in combined processes," Food Control, vol. 22, pp. 896-902, 2011.

[13] P. Dugo, I. Bonaccorsi, C. Ragonese et al., "Analytical characterization of mandarin (Citrus deliciosa Ten.) essential oil," Flavour and Fragrance Journal, vol. 26, no. 1, pp. 34-46, 2011.

[14] S. A. Vekiari, E. E. Protopapadakis, P. Papadopoulou, D. Papanicolaou, C. Panou, and M. Vamvakias, "Composition and seasonal variation of the essential oil from leaves and peel of a cretan lemon variety," Journal of Agricultural and Food Chemistry, vol. 50, no. 1, pp. 147-153, 2002.

[15] K. Hosni, N. Zahed, R. Chrif et al., "Composition of peel essential oils from four selected Tunisian Citrus species: evidence for the genotypic influence," Food Chemistry, vol. 123, no. 4, pp. 1098-1104, 2010.

[16] R. P. Adams, Identification of Essential Oil Components by Gas Chromatography/Quadrupole Mass Spectroscopy, Allured Publishing Corporation, Carol Stream, Ill, USA, 2001.

[17] J. L. Ríos and M. C. Recio, "Medicinal plants and antimicrobial activity," Journal of Ethnopharmacology, vol. 100, no. 1-2, pp. 80-84, 2005.

[18] B. M. Smallfield, J. W. Van Klink, N. B. Perry, and K. G. Dodds, "Coriander spice oil: effects of fruit crushing and distillation time on yield and composition," Journal of Agricultural and Food Chemistry, vol. 49, no. 1, pp. 118-123, 2001.

[19] H. Mhemdi, E. Rodier, N. Kechaou, and J. Fages, "A supercritical tuneable process for the selective extraction of fats and essential oil from coriander seeds," Journal of Food Engineering, vol. 105, no. 4, pp. 609-616, 2011.

[20] C. P. Des Gachons, C. Van Leeuwen, T. Tominaga, J. P. Soyer, J. P. Gaudillère, and D. Dubourdieu, "Influence of water and nitrogen deficit on fruit ripening and aroma potential of Vitis vinifera L cv Sauvignon blanc in field conditions," Journal of the Science of Food and Agriculture, vol. 85, no. 1, pp. 73-85, 2005.

[21] I. Bettaieb, N. Zakhama, W. A. Wannes, M. E. Kchouk, and B. Marzouk, "Water deficit effects on Salvia officinalis fatty acids and essential oils composition," Scientia Horticulturae, vol. 120, no. 2, pp. 271-275, 2009.

[22] M. M. Ahmad, S. U. Rehman, F. M. Anjum, and E. E. Bajwa, "Comparative physical examination of various citrus peel essential oils," International Journal of Agriculture and Biology, vol. 8, pp. 186-190, 2006.

[23] C. Blanco Tirado, E. E. Stashenko, M. Y. Combariza, and J. R. Martinez, "Comparative study of Colombian citrus oils by high-resolution gas chromatography and gas chromatography-mass spectrometry," Journal of Chromatography A, vol. 697, no. 1-2, pp. 501-513, 1995.

[24] R. Mebazaa, B. Rega, and V. Camel, "Analysis of human male armpit sweat after fenugreek ingestion: characterisation of odour active compounds by gas chromatography coupled to mass spectrometry and olfactometry," Food Chemistry, vol. 128, no. 1, pp. 227-235, 2011.

[25] D. L. Capone, K. Van Leeuwen, D. K. Taylor et al., "Evolution and occurrence of 1,8-cineole (Eucalyptol) in Australian wine," Journal of Agricultural and Food Chemistry, vol. 59, no. 3, pp. 953-959, 2011.

[26] W. Seo and H. H. Baek, "Identification of characteristic aroma-active compounds from water dropwort (Oenanthe javanica DC.)," Journal of Agricultural and Food Chemistry, vol. 53, no. 17, pp. 6766-6770, 2005.

[27] S. Selli and H. Kelebek, "Aromatic profile and odour-activity value of blood orange juices obtained from Moro and Sanguinello (Citrus sinensis L. Osbeck)," Industrial Crops and Products, vol. 33, no. 3, pp. 727-733, 2011.

[28] J. Muñoz-Bertomeu, R. Ros, I. Arrillaga, and J. Segura, "Expression of spearmint limonene synthase in transgenic spike lavender results in an altered monoterpene composition in developing leaves," Metabolic Engineering, vol. 10, no. 3-4, pp. 166-177, 2008.

[29] J. Degenhardt, T. G. Köllner, and J. Gershenzon, "Monoterpene and sesquiterpene synthases and the origin of terpene skeletal diversity in plants," Phytochemistry, vol. 70, no. 15-16, pp. 1621-1637, 2009.

[30] M. L. Lota, D. De Rocca Serra, F. Tomi, C. Jacquemond, and J. Casanova, "Volatile components of peel and leaf oils of lemon and lime species," Journal of Agricultural and Food Chemistry, vol. 50, no. 4, pp. 796-805, 2002.

[31] G. Flamini, M. Tebano, and P. L. Cioni, "Volatiles emission patterns of different plant organs and pollen of Citrus limon," Analytica Chimica Acta, vol. 589, no. 1, pp. 120-124, 2007.

[32] A. J. Poulose and R. Croteau, "Biosynthesis of aromatic monoterpenes: conversion of gamma-terpinene to p-cymene and thymol in Thymus vulgaris L.", Archives of Biochemistry and Biophysics, vol. 187, pp. 307-314, 1978.

[33] L. Sharon-Asa, M. Shalit, A. Frydman et al., "Citrus fruit flavor and aroma biosynthesis: isolation, functional characterization, and developmental regulation of Cstps1, a key gene in the production of the sesquiterpene aroma compound valencene," Plant Journal, vol. 36, no. 5, pp. 664-674, 2003.

[34] T. Shimada, T. Endo, H. Fujii, M. Hara, and M. Omura, "Isolation and characterization of (E)-beta-ocimene and 1,8 cineole synthases in Citrus unshiu Marc," Plant Science, vol. 168, no. 4, pp. 987-995, 2005.

[35] F. Bakkali, S. Averbeck, D. Averbeck, and M. Idaomar, "Biological effects of essential oils-a review," Food and Chemical Toxicology, vol. 46, no. 2, pp. 446-475, 2008.

[36] S. Burt, "Essential oils: their antibacterial properties and potential applications in foods," International Journal of Food Microbiology, vol. 94, no. 3, pp. 223-253, 2004.

[37] S. Santoyo, S. Cavero, L. Jaime, E. Ibañez, F. J. Señoráns, and G. Reglero, "Chemical composition and antimicrobial activity of Rosmarinus officinalis L. essential oil obtained via supercritical fluid extraction," Journal of Food Protection, vol. 68, no. 4, pp. 790-795, 2005.

[38] A. P. Longaray Delamare, I. T. Moschen-Pistorello, L. Artico, L. Atti-Serafini, and S. Echeverrigaray, "Antibacterial activity of the essential oils of Salvia officinalis L. and Salvia triloba L. cultivated in South Brazil," Food Chemistry, vol. 100, no. 2, pp. 603-608, 2007.

[39] A. H. Ebrahimabadi, E. H. Ebrahimabadi, Z. Djafari-Bidgoli, F. J. Kashi, A. Mazoochi, and H. Batooli, "Composition and antioxidant and antimicrobial activity of the essential oil and extracts of Stachys inflata Benth from Iran," Food Chemistry, vol. 119, no. 2, pp. 452-458, 2010.

[40] R. Kotan, S. Kordali, and A. Cakir, "Screening of antibacterial activities of twenty-one oxygenated monoterpenes," Zeitschrift 
fur Naturforschung Section C, vol. 62, no. 7-8, pp. 507-513, 2007.

[41] A. C. Goren, F. Piozzi, E. Akcicek et al., "Essential oil composition of twenty-two Stachys species (mountain tea) and their biological activities," Phytochemistry Letters, vol. 4, no. 4, pp. 448-453, 2011.

[42] H. J. D. Dorman and S. G. Deans, "Antimicrobial agents from plants: antibacterial activity of plant volatile oils," Journal of Applied Microbiology, vol. 88, no. 2, pp. 308-316, 2000.

[43] R. Randrianarivelo, S. Sarter, E. Odoux et al., "Composition and antimicrobial activity of essential oils of Cinnamosma fragrans," Food Chemistry, vol. 114, no. 2, pp. 680-684, 2009.

[44] A. I. Hussain, F. Anwar, P. S. Nigam et al., "Antibacterial activity of some Lamiaceae essential oils using resazurin as an indicator of cell growth," LWT-Food Science and Technology, vol. 44, pp. 1199-1206, 2011.

[45] Y. Inoue, A. Shiraishi, T. Hada, K. Hirose, H. Hamashima, and J. Shimada, "The antibacterial effects of terpene alcohols on Staphylococcus aureus and their mode of action," FEMS Microbiology Letters, vol. 237, no. 2, pp. 325-331, 2004.

[46] J. M. Schmidt, J. A. Noletto, B. Vogler, and W. N. Setzer, “Abaco bush medicine: chemical composition of the essential oils of four aromatic medicinal plants from Abaco Island, Bahamas," Journal of Herbs, Spices and Medicinal Plants, vol. 12, no. 3, pp. 43-65, 2006. 

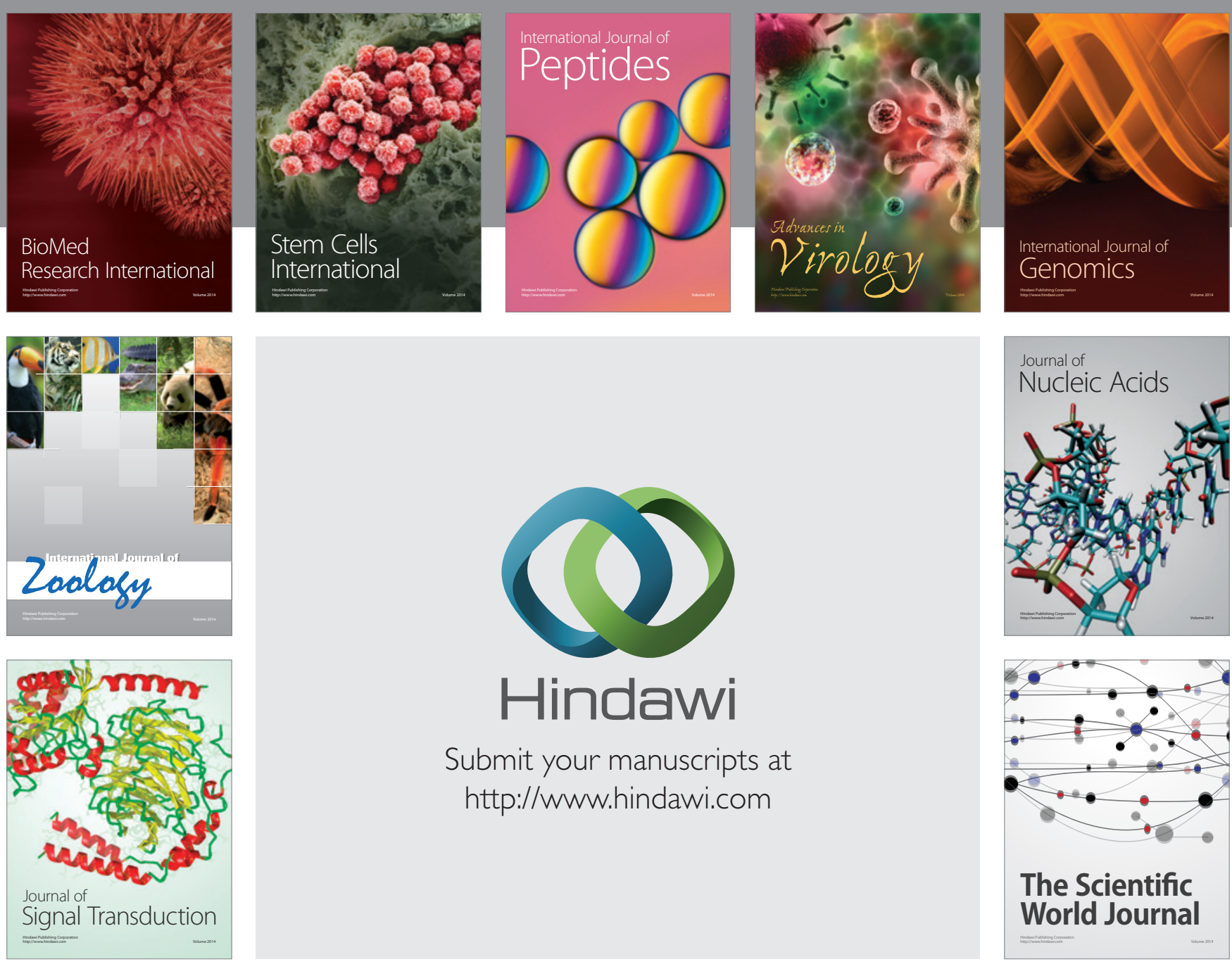

Submit your manuscripts at

http://www.hindawi.com
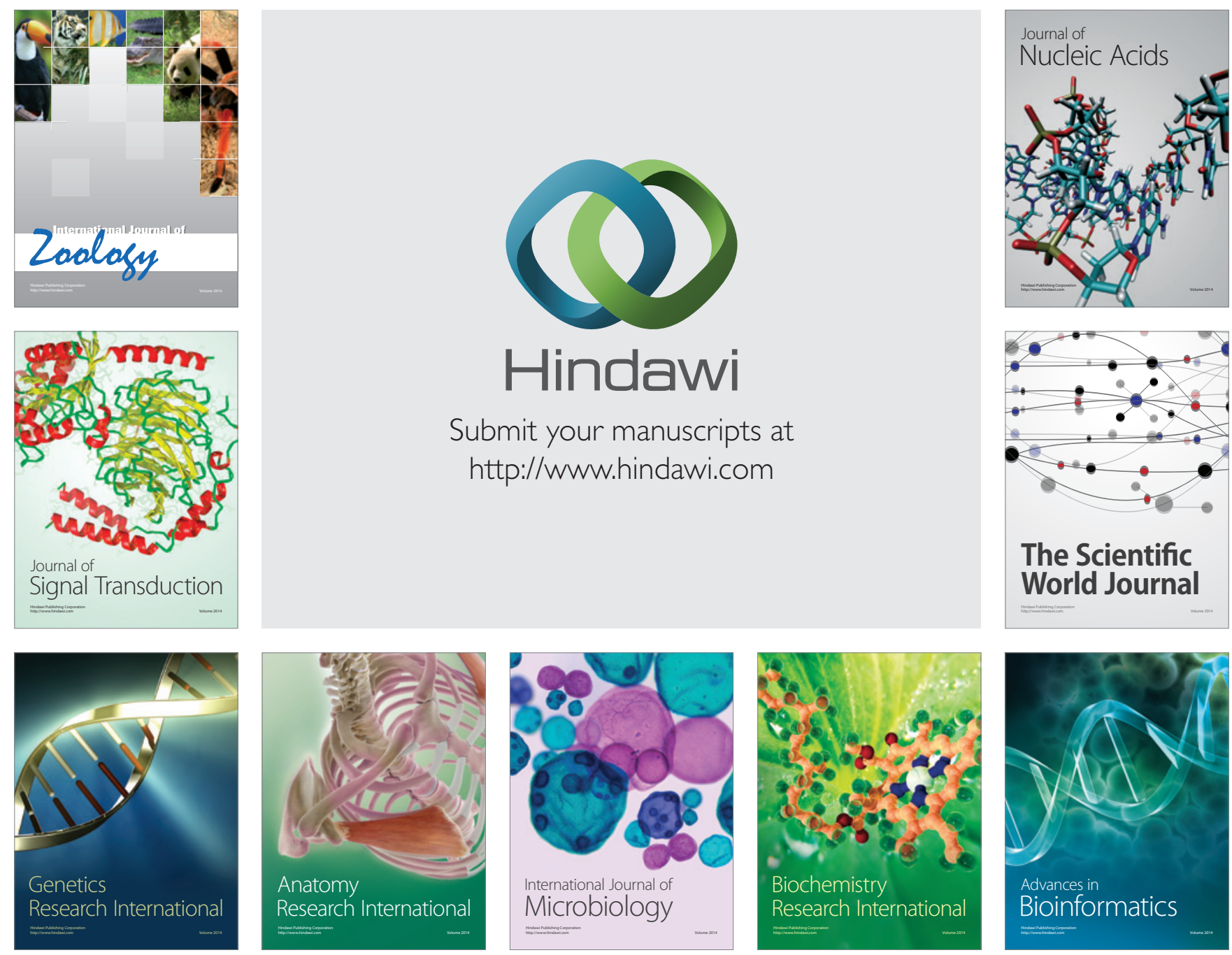

The Scientific World Journal
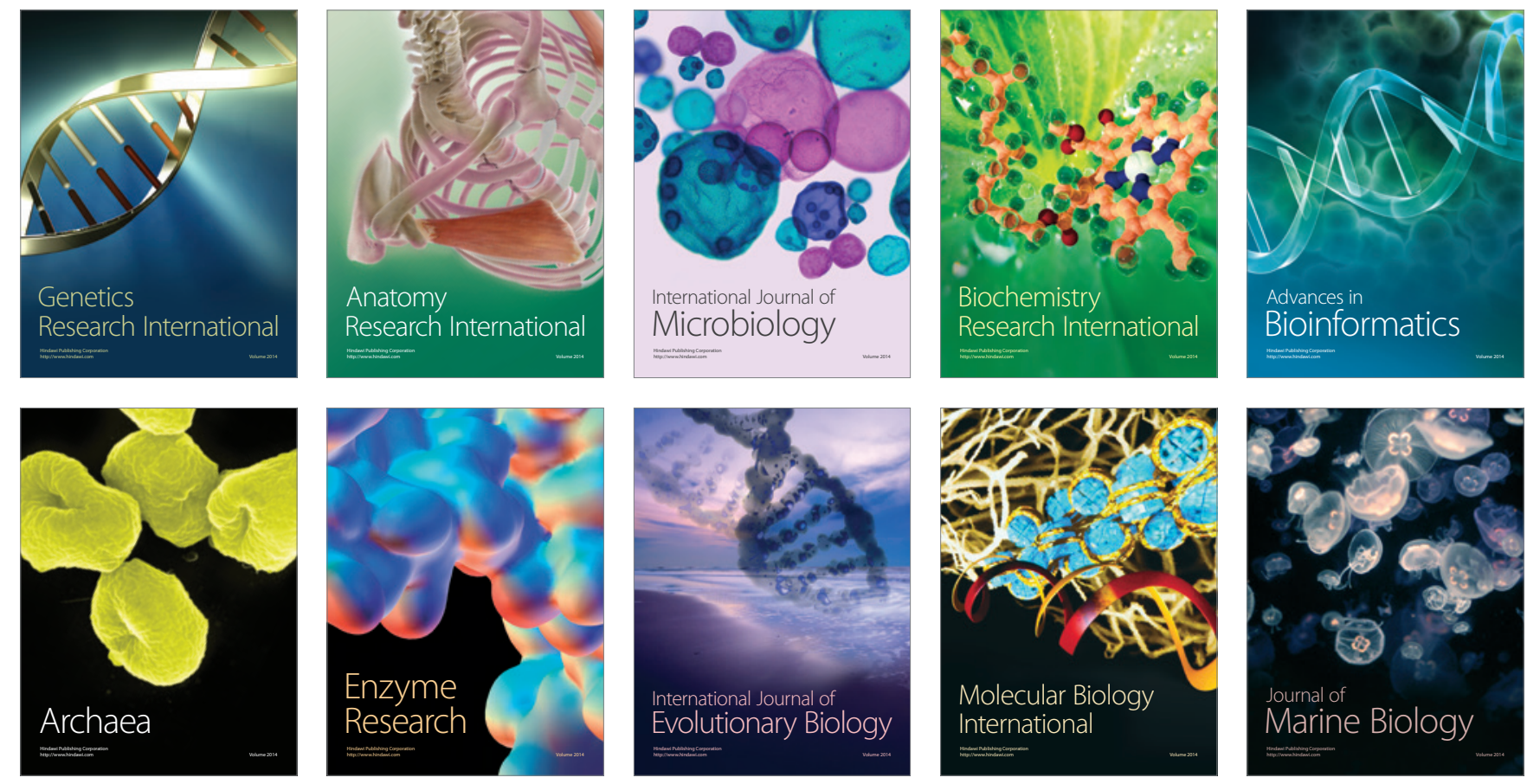